\title{
ANÁLISIS HISTÓRICO DE LA SITUACIÓN DE LAS ENFERMERAS Y MATRONAS EN ESPAÑA. UNA PERSPECTIVA GLOBAL ${ }^{1}$
}

\author{
Carmen González Canalejo²
}

Resumen: Al ser la enfermería y la matronería profesiones ejercidas mayoritariamente por mujeres en todos los países del mundo, nos hemos centrado en el análisis histórico de su práctica, poniendo el foco en los factores que han influido en la desigualdad de oportunidades para optar a puestos de gestión sanitaria y, en un menor reconocimiento científico de su práctica. Conocer la seña de identidad femenina y feminista de las que optaron por estas profesiones, valorar su estatus laboral y analizar las reminiscencias patriarcales que hoy día se mantienen en el ámbito de la salud, es el objeto de este estudio.

Palabras clave: Enfermeras, matronas, invisibilidad, cuidados de salud, androcentrismo, sistema sanitario.

Title in English: Historical Analysis of the Situation of Nurses and Midwives in Spain. A Global Perspective

Abstract: Globally, nursing and midwifery are professions carried out mainly by women. This historical analysis of the praxis of nurses and midwives focuses on the factors that contribute to discrimination against them, both in terms of opportunities to attain health management jobs, and in terms of scientific recognition for their praxis. The goals of this study are, therefore, to determine the female identity traits and the feminist ideas of those who opt for these professions, to evaluate their work status, and to analyse the patriarchal traditions that still prevail nowadays in the area of health.

Key words: Nurses, midwives, health care, invisibility, androcentricity, health system.

\section{Origen y antecedentes}

Los estudios historiográficos sobre género señalan que la práctica de los "cuidados de salud"3 no es algo nuevo, pues la sanación y la promoción de la vida han

\footnotetext{
${ }^{1}$ Fecha de recepción: 30/09/2013

Fecha de aceptación: 02/12/2013

${ }^{2}$ Profesora Titular de Universidad, Departamento de Enfermería, Fisioterapia y Medicina, Universidad de Almería, España; $₫$ canalejo@ual.es.

${ }^{3}$ Este término ha sido adoptado por las teóricas de la enfermería para definir la naturaleza de su práctica así como el fundamento científico que la sustenta. Tanto las mujeres que accedieron al título de
} 
sido ejercidas, tanto por varones como por mujeres, desde los tiempos más remotos (Colliére 1993: 5 y ss.). Sin embargo, en el ámbito privado y doméstico han sido principalmente las mujeres quienes han llevado a cabo el arte de partear y el cuidado, quedándose a su cargo los enfermos del grupo familiar (Santo-Tomás 1995: 3-129). Entre otros factores, porque las mujeres siempre han preferido a la hora del parto ser atendidas por especialistas femeninas.

De acuerdo con los estudios que mantienen el incremento de la participación laboral femenina a raíz de la industrialización (Borderías et al. 1994: 54-59), existen tres factores determinantes para que el Estado Contemporáneo institucionalizase a las matronas y enfermeras a lo largo de la segunda mitad del siglo XIX. Dichos factores se concretan en la necesidad de las naciones de incorporar a las mujeres al mundo productivo, en general. Pero, desde principios del siglo XX, también cabe citar el deseo de una independencia económica y personal al que aspiraban las mujeres. Por último, hubo necesidad, por parte de la Institución Benéfico-asistencial del último cuarto del siglo XIX y primer tercio del XX, de contratar a un mayor número de mujeres sanitarias para dar respuesta al grave problema de salud materno-infantil derivada de la pobreza social (González 2007).

Para comprender el contexto en el que se desarrolla el trabajo de las matronas y enfermeras contemporáneas es necesario situarnos en el marco de su práctica, basada en el cuidado de salud y la promoción de la vida. Actividades que las mujeres han realizado bien de modo informal, a través de la atención a los enfermos en el ámbito privado, bien de modo "oficial” en el ámbito público, a través de la asistencia benéfica o por iniciativa particular (Alberdi 1998: 63-73). En el año 1857 la Ley Moyano dio acceso a las mujeres a los estudios de matrona, convirtiéndose en las primeras españolas que pisaron las facultades de medicina restringidas hasta entonces a los varones. Teniendo en cuenta el peso que representaban los roles sexuales como obstáculo en el acceso a la educación de la mujer, no es de extrañar que la postura más generalizada en el orden patriarcal de aquella época fuera considerar, a los estudios de matrona, más 
“adecuados" al sexo femenino (Martínez y Ortiz 1998: 603) igual que ocurriría con los de enfermería. Desde la década de los años setenta del siglo XIX se documentan numerosos proyectos educativos promovidos por mujeres, quienes impulsaron el acceso femenino a estas profesiones con el rasgo característico de que no les fue reconocida la autoría de tales proyectos.

En el año 1896 se creó la primera escuela de enfermeras profesionales en España, tras la reforma planteada por Concepción Arenal unos años antes (Siles 1995: 169 y ss.). Hasta la segunda década del siglo XX, al menos hubo dos proyectos más de creación de escuelas para los estudios de matrona y, como mínimo, tres escuelas más de enfermería. Sin embargo, algunos de estos proyectos fracasaron por las enormes trabas y dificultades impuestas a las mujeres. El mundo sanitario era el reflejo de una sociedad patriarcal donde médicos del orden conservador y años más tarde, los practicantes, se opusieron de forma contundente a que las mujeres ocuparan las aulas en las facultades de medicina y cobraran protagonismo científico en el ámbito sanitario (González 2006).

Muchos facultativos percibieron tal acontecimiento como un desafío ya que la mujer no era considerada sujeto de derechos. Veían en ellas a unas competidoras, a la vez que estaban convencidos de que cierto status profesional pertenecía exclusivamente a los varones. En opinión de otros, la cuestión no debía preocupar, al comprobar el escaso éxito que el acceso educativo de las primeras aspirantes al título de matrona había tenido en España (Álvarez 1988: 171).

Hasta el año 1904, fecha en la que hubo un proyecto de reforma sanitaria, las mujeres no pudieron entrar en las facultades para estudiar la carrera de "practicante". Estudios que, por cierto, tuvieron una corta existencia para las mujeres al desaparecer en la primera etapa del franquismo. Sin embargo, cabe destacar que hubo intentos por parte de ellas de acceder a la profesión de practicante, al menos desde el año 1900. Capel apunta que en esta fecha ya hubo algunas aspirantes matriculadas de forma "oficiosa", a pesar de que legalmente no les estaba permitido. De modo que sus títulos no fueron legalizados hasta varios años después (Capel 1982: 435 y ss).

Un aspecto característico del debate mantenido en los años de cambio de siglo sobre lo que llegó a denominarse "la cuestión de la mujer" era si al incorporarse estas al 
mundo productivo, olvidarían "las labores propias de su sexo". El trabajo doméstico, el cuidado familiar, incluidos los ancianos del grupo y la educación de los hijos eran el imperativo predominante en la vida de las que optaron por el mundo laboral remunerado. La condición que emanaba de esta premisa era que las mujeres se incorporarían a la esfera productiva, siempre y cuando no olvidaran la función doméstica y de maternidad que el orden social les había asignado (Blanco et al. 2002). A pesar del freno que puso el sector sanitario conservador y la propia Iglesia, el número de aspirantes al título fue en ascenso a partir de la segunda década del siglo XX. Aún así, tanto enfermeras como matronas representaban una ínfima minoría con respecto a un panorama sanitario mayoritariamente masculino, hasta la llegada de la Segunda República que impulsó a la cotidianeidad del mundo del trabajo a las mujeres (Domínguez 1986).

No obstante, una cosa era la incorporación al mundo laboral y, otra muy distinta, el reconocimiento de la igualdad de derechos. El discurso androcéntrico predominante en el campo de la salud desde finales del siglo XIX cuyas reminiscencias alcanzan hasta nuestros días, ha tenido como característica la desautorización del conocimiento de quienes ejercen estas profesiones al considerarlas "auxiliares" respecto a la medicina, cuya práctica ha sido ejercida hasta hace escasamente cinco años por varones, predominantemente.

\section{La inestimable contribución a la salud de la ciudadanía}

El eje principal de la práctica enfermera continúa actualmente siendo los cuidados. Definir qué es el "cuidado" no es tarea fácil dado que reviste un sin fin de actividades encaminadas al mantenimiento de la salud y la preservación de la vida. En los años cincuenta del pasado siglo, Virginia Henderson, una de las máximas exponentes que ha dado el campo de la enfermeríaindica en su obra, Textbook of the Principles and Practise of Nursing, la variedad y complejidad del trabajo de las enfermeras (Harmer y Henderson 1955: 10). En la citada obra, distintos informes revelan que las enfermeras llevan a la práctica más de 500 actividades distintas en la 
asistencia hospitalaria, domiciliaria, industrial y privada. Características que pueden extenderse al campo de la matronería, donde la práctica no se limita a la mera asistencia técnica del parto, sino a una multitud de actividades que giran en torno a las mujeres asistidas y los neonatos.

De modo que tanto la enfermería como la matronería son prácticas complejas. Se trata en ambos casos de actividades tremendamente minuciosas que implican operaciones de análisis razonado, de habilidades técnicas, afectivas y de comunicación. Aspectos estos que forman un difícil entramado y que se conjugan en cada una de las tareas que tratan de cubrir las necesidades de un paciente y van más allá de la asistencia en la enfermedad. La característica esencial estriba en una atención holística y humanizada a la persona asistida cuyas necesidades de salud no son estáticas sino impredecibles que, a veces, requieren urgencia y experiencia en la atención. Se trata sobre todo, de combinar la observación y el conocimiento con una relación humana, directa con la persona atendida, llegando a convertirse en una relación "cuerpo a cuerpo", entre las cuidadoras y los asistidos. Según Henderson para una persona sana, la respiración, por ejemplo, no requiere esfuerzos. La enfermera que coloca al paciente con una fractura de costillas en una postura cómoda que le permita una respiración de calidad o la que trabaja con un paciente conectado a un respirador, está ejecutando funciones complejas. Porque no se trata mecánicamente de conectar a la persona afectada a una máquina o canalizarle una vena que emigre desde el brazo hasta la aurícula del corazón, lo cual también requiere destreza y conocimiento. Se trata esencialmente de que el paciente acepte la máquina, la tolere y sea compatible con la vida y la dignidad humana, pues la enfermera estará pendiente de que el sufrimiento no prive a la persona de su dignidad como ser humano que es.

Y este abanico de actividades del día a día dirigidas de manera permanente hacia las personas con requerimientos de cuidados de salud, no es sencillo cuando hay que ajustarlo a las demandas individuales. Trabajo que no está lo suficientemente valorado ni aún en los países occidentalizados donde existe un déficit de enfermeras, pues la mayoría abandona el empleo tras apenas una década después de obtener el título en la Universidad. Un caso significativo es el de EEUU donde las asociaciones enfermeras, 
llevan años concienciando al gobierno norteamericano de la necesidad de un mayor esfuerzo hacia la revalorización social de este colectivo. Inglaterra, Suecia y Francia ante el déficit de enfermeras autóctonas, están importando a jóvenes titulados de otros países cuya cantera, mayoritariamente femenina, proviene de España. A ello también favorece la amplia formación con la que se capacita al alumnado español, al avanzado sistema de salud pública y al desempleo que la crisis económica ha provocado en los últimos años en el seno de la sanidad española (González 2012: 3).

Estudios publicados desde finales de los años ochenta han venido mostrando con indicadores cuali-cuantitativos, la elevada presencia de mujeres que trabajan en los sistemas sanitarios en más de una treintena de países de todo el mundo, siendo éstas la base de la pirámide en la que se sostiene el sistema mundial de la salud (García 1988; Pizurki 1988; Domínguez 1993: 223). Por otra parte, fuentes del Ministerio de Educación y Ciencia señalan que el ingreso femenino en las carreras del campo de la salud en el año 2002 era de un 71\%, porcentaje que siguió en los sucesivos años en aumento y que se mantiene actualmente por encima de la media de los países del entorno europeo.

En Europa se está prestando una mayor atención a la profesión de enfermería y la matronería por ser este colectivo un número muy representativo de mujeres quienes las ejercen. A escala mundial, según informes de la OMS, el $87 \%$ del colectivo enfermero está formado por mujeres y, en el caso de la matronería, la estadística raya prácticamente en el 98\% de los casos (Pizurki 1988: 63).

Sin embargo, esta representación tan numerosa contrasta con la invisibilidad de la autoridad femenina, del saber científico que encierra su práctica. Estudios de género de investigadoras especializadas en el tema sanitario han puesto en evidencia el androcentrismo que ha impregnado, desde tiempos históricos, el campo de la salud y cómo el saber científico de las mujeres se ha obviado en la investigación sanitaria. Enfermeras, matronas, sanadoras, y las médicas también, han escrito libros y tratados con rigurosidad científica desde épocas muy antiguas los cuales, con frecuencia, fueron copiados con autoría masculina (Ortiz 2006; Miqueo et al. 2003: 195-216). Ellas han sido testimonios directos del allanamiento de sus funciones en el ejercicio profesional y 
del cuestionamiento de su capacidad para el ejercicio sanitario, cuestiones que han sido denunciadas de forma abierta y explícita por las primeras que accedieron al trabajo en los domicilios, en los dispensarios y en las salas de hospital. Líderes universalmente reconocidas como Florence Nightingale y también las matronas, enfermeras y practicantas locales han propuesto intervenciones reparadoras de la tremenda desigualdad social que han sido recogidas en testimonios directos, escritos y orales (González 2006).

Hay estudios que han demostrado que la supervivencia profesional de las que optan por las carreras sanitarias puede resultar difícil con independencia de su categoría profesional, porque las jefaturas de los servicios médico-quirúrgicos, principalmente de los hospitales y determinados espacios sanitarios, han sido ocupadas tradicionalmente por varones. Un ejemplo bastante significativo es el que concierne a las especialidades quirúrgicas, donde los informes de la propia Confederación Estatal de Sindicatos Médicos, muestra que las cirujanas españolas no suman más del $10 \%$ en algunas especialidades quirúrgicas, a la vez que éstas describen los quirófanos como lugares emblemáticamente masculinizados a los que señalan como "espacios altamente competitivos y rudos" (Ceballos 2002: 8).

En definitiva, la mayoritaria proporción, participación y demostrada contribución social de las mujeres en los sistemas sanitarios de los distintos países a lo largo del tiempo, no ha sido suficiente para que éstas estén presentes en puestos de relevancia en la gestión y administración sanitarias, en las mismas condiciones que los varones.

\section{La transformación ${ }^{4}$}

El cambio social más significativo en los colectivos de enfermeras y matronas fue la ruptura del discurso "doméstico" de los cuidados producto de un patriarcado en el

\footnotetext{
${ }^{4}$ Para este punto partimos de fuentes primarias existentes en los Colegios Oficiales profesionales, el INE, la Delegación de Sanidad y, el Anuario Estadístico de España.
} 
ámbito de la salud que adscribía a las que eligieron estas profesiones, a una práctica meramente técnica, carente de razonamiento y de análisis crítico. Coincidiendo con la reivindicación feminista de autodeterminación en la etapa política de la Transición las enfermeras decidieron, a partir del año 1977, rechazar actividades de subordinación y ayuda al médico para centrarse en su auténtica actividad: la ayuda y cuidado a la ciudadanía. Esta reivindicación fue femenina y feminista, pues los ATS varones no secundaron la huelga producida en aquellos años cuyo sello llevaba implícito el anhelo de la autodeterminación (Vigil 1996: 40). El logro de aquel entonces se vio retroalimentado por el empuje democrático que favoreció un "despegue" en el campo del conocimiento propio de estas profesiones y ha repercutido en un notable aumento de la calidad sanitaria que hoy día ha sido reconocida internacionalmente. La reivindicación explícita, tal y como hemos venido indicando, estriba en que sus protagonistas no querían ser tutorizadas por los médicos, ni realizar actividades delegadas de los mismos, sino colaborativas e interdisciplinares. El derecho a ejercer lejos del control médico ha sido un antiguo anhelo desde que se configuraron las primeras asociaciones femeninas matronales, en los años veinte del pasado siglo y se acusó aún más durante el franquismo. A partir del año 1985, la aspirada autonomía se ha venido justificando razonadamente desde los campos asistencial y educativo. En esta última fecha, la mayoría de las escuelas donde se cursaban estos estudios estaban bajo el control de los médicos. Mientras tanto, en Francia, Inglaterra y otros países del entorno el aspecto académico y del conocimiento era gestionado mayoritariamente por las propias enfermeras o matronas desde hacía varias décadas.

El derecho de autogestión de las matronas y enfermeras no solo se ha debatido en el ámbito académico. También ha marcado amplias discusiones en los servicios hospitalarios de la sanidad pública, en centros de salud, las consultas domiciliarias, las de atención a pacientes crónicos, servicios de atención a las gestantes, consulta del niño sano y de autogestión de las competencias de las recién creadas enfermeras de enlace 5 .

\footnotetext{
${ }^{5}$ La enfermera de enlace es una figura reciente, pues ha sido creada en el año 2004 en los sistemas de salud de algunas comunidades autonómicas como es el caso de Andalucía. La complejidad del entramado sanitario ha hecho necesario la puesta en marcha de esta figura creada para ayudar al usuario a acceder al sistema, según las necesidades de salud del paciente.
} 
Sin embargo, para conseguir la igualdad de oportunidades en los cargos de gestión sanitaria, todavía falta camino por recorrer. El camino allanado durante décadas ha dado como resultado el hecho de que un sustancial número de servicios de salud esté siendo gestionado satisfactoriamente por matronas y enfermeras. Ahora bien, todavía queda camino por recorrer para conseguir plena igualdad en el plano de la realidad. Si bien es verdad que actualmente no se cuestiona la capacidad de las mujeres para ejercer en cualquiera de las disciplinas sanitarias y los derechos laborales están garantizados por ley, se puede constatar que la gestión de los servicios de salud se ha convertido en un estatus de tradicional arraigo masculino a cuyos miembros no les ha importado que las mujeres sanitarias accedan a puestos intermedios, dado que en los niveles más elevados siguen estando mayoritariamente ocupados por los varones de la sanidad. Los mejor pagados y con mayor prestigio continúan siendo quienes desempeñan la medicina o algunas de sus ramas, a la vez que mantienen el control de los espacios más tradicionales como los hospitales y los puestos de alta gestión, donde se toman las últimas decisiones en materia de salud pública (Lillo 2002: 56).

El hecho de que en España el cargo de máxima autoridad sanitaria lo ostente una mujer ministra no significa en modo alguno que el poder esté repartido de forma igualitaria entre los hombres y mujeres de este campo. Al contrario de lo que cabría imaginar, tal y como se desprende del análisis de los datos obtenidos para este estudio, a pesar de que las mujeres sobrepasan en número al de los varones, en la mayoría de las ramas sanitarias, éstas no participan en las cotas más altas de poder donde el panorama refleja una realidad bien distinta. A este respecto, son bastante ilustrativos los datos de fuentes oficiales provenientes de los colegios profesionales andaluces donde los cargos presidenciales de los colegios de medicina, odontología, fisioterapia, farmacia y en menor caso, enfermería, son ocupados mayoritariamente por varones ${ }^{6}$. Si ascendemos en la escala en los puestos de gestión pública, en relación a los delegados provinciales

\footnotetext{
${ }^{6}$ En el caso de Andalucía, en el año 2004, el 80\%, de los cargos eran ocupados por varones, a pesar de ser una de las autonomías que más se ha esforzado en los últimos años en la consecución de la igualdad de oportunidades. A excepción de los colegios de enfermería y matronería donde se observa mayor grado de paridad, los miembros que presidían en esta fecha los Colegios de farmacéuticos, estomatólogos, fisioterapeutas y medicos corresponde a varones, en la mayoría de las provincias. Fuente: Colegios Oficiales provinciales de médicos, estomatólogos, enfermería, farmacéuticos y fisioterapia de Andalucía, accesible a través de las webs de los correspondientes colegios.
} 
de salud, asesores y consejeros, la casi totalidad han sido varones hasta el año 2004. A partir de esta fecha que marca el inicio de la política de igualdad en los cargos públicos, los equipos provinciales y autonómicos cada vez fueron integrando a un mayor número de mujeres en Andalucía. En cuanto a las direcciones de enfermería hospitalaria, se produce lo que se denomina el "efecto de la pirámide invertida", dato que llama, si cabe, aún más la atención al ser éste un sector mayoritariamente femenino donde de cada cinco direcciones, cuatro son ocupadas por enfermeros y sólo una es enfermera. Sin embargo, en la Atención Primaria los cargos de mayor responsabilidad están más repartidos entre hombres y mujeres que en las áreas hospitalarias. En este caso, existe un reparto más paritario. Aquí el organigrama sanitario no está tan estratificado como en los hospitales, existiendo solamente dos figuras: Directores/as y Coordinadores/as y están repartidos entre varones y mujeres sin distinción de categorías profesionales, es decir, los ocupan tanto médicos/as como enfermeras/os, en torno al $50 \%{ }^{7}$.

En definitiva, los datos revelan que las mayores cotas de poder en el ámbito de la sanidad pública se concentran en los espacios hospitalarios y en los puestos de mayor representación, estatus social y, nivel económico y que dichas cotas están todavía representadas mayoritariamente por varones. Concretamente, en la cúpula de la medicina, enfermería-matronería, farmacéuticos, fisioterapeutas y odontólogos, las mujeres, a pesar de ser quienes mayoritariamente ostentan el trabajo sanitario, están lejos, todavía, de alcanzar los puestos de gestión de alto nivel en cada una de estas ramas. Sin embargo, desde el impulso de la Ley de Igualdad de Oportunidades del año 2004 y el hecho de que una mayoría de mujeres ejerza como médicas de familia, además de la marcada existencia de enfermeras en los espacios de Atención Primaria ha contribuido, en el caso concreto de Andalucía, en favor de la paridad en la gestión concretamente en éste área de atención pero no en el área hospitalaria tradicional, donde el peso del fenómeno androcentrico está todavía muy presente.

\footnotetext{
${ }^{7}$ Dada la ausencia de estadísticas oficiales en relación a las direcciones de enfermería hospitalaria y de Atención Primaria, para ilustrar este aspecto hemos recurrido a fuentes orales colegiadas a nivel autonómico andaluz.
} 


\section{Conciencia y estrategia de cambio}

El trabajo de algunas historiadoras sobre el tardío acceso de las mujeres de Europa, en general, al mundo de la educación es bastante ilustrativo (Capel 1982; Ballarín 2001). Dentro de lo tardío que fue este acceso en España, la historiografía de género señala que las matronas fue el colectivo femenino de las disciplinas sanitarias que más tempranamente accedieron a la Universidad, en el año 1857 (González 2006: 26). Después, las siguieron las primeras aspirantes a medicina matriculándose en un grupo de alumnas la Facultad de Barcelona quienes acabarían como médicas a principios de 1880 (Flecha 2001: 217). Ya en los años finiseculares, se incorporaron las enfermeras a raíz de la creación de la primera escuela profesionalizada en Madrid, al calor de la Institución Libre de Enseñanza, en el año 1896 (Domínguez 1986: 315).

Lo que podría ser una efemérides no lo es. A día de hoy, son más del $73 \%$ las mujeres que acceden a los estudios de las Ciencias de la Salud, es decir, es una presencia "abrumadora", tal y como ya se ha señalado (De la Rica y Brinduesa 2010: 25). Efectivamente, nunca antes en la historia, las mujeres que han ejercido en este sector han estado tan preparadas para acceder al conocimiento, pero a pesar de la "abrumadora" mayoría de las que han finalizado los estudios de matronería y/o de enfermería, tienen serias dificultades para acceder a los estudios de doctorado, dentro de su propio campo disciplinar. Entre otros factores, debido al escaso tiempo transcurrido desde que estas carreras pasaran de la Diplomatura al Grado gracias a la Convergencia con Europa. Ello significa que apenas exista una media docena de programas de tercer ciclo específicos de estos estudios en España a los que ellas puedan acceder, y muy pocas las enfermeras docentes con sexenios homologados que puedan dirigir tesis doctorales por las duras dificultades a las que las investigadoras se han tenido que sobreponer en el mundo académico. Se da la circunstancia de que en las últimas décadas, los gobiernos ya sean de izquierdas o de derechas han negado los estudios de tercer ciclo en estas titulaciones donde la proporción de alumnas es cinco veces superior 
al número de varones matriculados. Fenómeno que cabe interpretarlo en clave de género. La situación de discriminación tanto en la promoción laboral de las que eligen estas carreras como en el ámbito académico es alarmante. Al hilo de lo planteado, a pesar de ser la licenciatura y doctorado en enfermería y matronería una de las reivindicaciones históricas de quienes ejercen estas profesiones, las expectativas que actualmente se vislumbran están, si cabe, más ensombrecidas. Si se cumplen los acuerdos de homologación establecidos desde el inicio del proceso de la Convergencia con Europa, se habrá podido salvar esta discriminación histórica, cuya desigual oportunidad afecta a sectores profesionales y académicos ocupados mayoritariamente por mujeres, no solo en España ${ }^{8}$, sino en todos los países de la Unión Europea.

Otra de las dificultades de las que se ha tomado conciencia es el desarrollo de lo que hoy día se considera calidad de vida profesional. Dichas dificultades son comunes a las que plantea la incorporación laboral de las mujeres en general, y se basa en cómo éstas compaginan la vida laboral y la vida familiar. Ya sean médicas, matronas, enfermeras o farmacéuticas, da igual la categoría profesional ejercida, las mujeres sanitarias han desarrollado estrategias para poder compaginar el trabajo con la vida familiar. Camino que continúa lleno de dificultades al ser los varones españoles los que dedican menos horas al trabajo familiar, cuya cifra es significativamente inferior al resto de los países del entorno (Ramos y Romo 1998).

Para vencer estas dificultades, las profesionales de la sanidad se valen de estrategias como cambiar los turnos de trabajo para combinar la jornada laboral con las gestiones familiares de urgencia (cuidar a un hijo o familiar enfermo, trámites o gestiones del hogar, etc.); preferencia de turnos de "noches fijas" que les permiten continuar con las actividades domésticas durante el día. En otras ocasiones buscan redes de apoyo familiares. Suelen acudir principalmente a madres, hermanas o amigas para que les recojan a los hijos del colegio o les ayuden en las tareas domésticas. Todo ello

\footnotetext{
${ }^{8}$ En lo que a España se refiere, según fuentes informatizadas de los propios centros, más de la mitad de las 97 facultades de enfermería dependientes de las universidades públicas que se encuentran repartidas por el territorio español, 29 de ellas tienen a la cabeza una directora, 14 están dirigidas por varones y del resto, no se muestra ningún dato.
} 
sin olvidar que la mujer-enfermera o sanitaria, en general, que trabaja en la esfera pública, también ejerce esta cualificación en el seno familiar a la que todos los miembros: madre, tíos, abuelos, hermanos, hijos o sobrinos, la designan como "idónea" para atender y cuidar al resto de los componentes. A menudo, el entorno familiar se convierte en una continuación de la vida laboral, donde se suma, a las cargas de trabajo, el cuidado de salud del resto de los miembros de la familia. Ello ha favorecido, según las estadísticas de salud laboral en el ramo sanitario, que las enfermeras sufran estrés en mayor medida que el resto de los grupos sanitarios profesionales (Moreno 2002: 18).

Un último problema del que se ha tomado conciencia colectiva deriva de los estereotipos hacia las enfermeras derivados de la ideología de género. De todas las profesiones sujetas a dichos estereotipos, la de enfermería aparece como una de las más afectadas, pues según los clichés derivados de la mentalidad patriarcal las enfermeras, en general, han sido consideradas como un elemento secundario dependiente de los médicos. Al papel subordinado hay que sumar el hecho de que a menudo se las contempla como un objeto sexual al que ha contribuido un determinado tipo de cine, series televisivas y algunos medios de comunicación que han derivado en mostrar una imagen de mujer carente de conocimientos y de análisis crítico. Las protagonistas de estos prototipos desarrollan un rol de auxiliadoras de los médicos, en lugar de cuidadora de los pacientes $\mathrm{y}$, raramente, se las muestra desempeñando un papel social como integrante de un equipo con unas señas de identidad, criterio científico y autonomía profesional propia.

No obstante, cabe añadir que la conciencia de esta situación está articulando medidas de "resolución" entre las enfermeras. Existe un debate actual en el mundo occidental donde se está haciendo un mayor hincapié en ir modificando esta imagen negativa. A este respecto, en informes presentados al Congreso Internacional de Enfermería (CIE) celebrado en Ginebra en el año 2003, es significativa la política activa que los países del Norte de Europa, EE. UU y en menor medida en España, están llevando a cabo para neutralizar la imagen estereotipada de las enfermeras (CIE 2003). La puesta en marcha de programas para apoyar la igualdad de oportunidades e incentivar la motivación y visibilidad de las mismas en el ámbito sanitario y académico, 
tiene como fin difundir las aportaciones de las mujeres a este campo y garantizar su permanencia y promoción profesional. Así pues, el verdadero desafío en el presente tiene dos vertientes paralelas. Una, depende del proceso de autonomía, entendida como la capacidad de autogestión en la toma de decisiones propias de la disciplina del cuidado, basada en la participación paritaria de hombres y mujeres. La otra vertiente de actuación estriba en recordar el papel social que han realizado las matronas y enfermeras en beneficio de la salud pública a lo largo de la historia. A este respecto, las hemerotecas y los archivos hablan por sí solos sobre la actividad divulgativa y profiláctica de quienes han ejercido estas profesiones y que se siguen llevando a cabo en las políticas de salud a escala mundial.

A modo de conclusión cabe señalar que, a la hora de valorar los cambios significativos de las que ejercen la enfermería y matronería en los últimos treinta años y de las repercusiones favorables derivadas de la Ley de Igualdad promulgada en el año 2004, a día de hoy se mantienen desigualdades en relación con tres aspectos, principalmente. El primer aspecto está relacionado con el escaso reconocimiento científico de las que ejercen estas profesiones, en su mayoría mujeres cuya población laboral asciende a más de un $80 \%$ tanto en España como en Europa; el segundo aspecto es el relacionado con el difícil acceso a los estudios de tercer ciclo y desarrollo académico dentro de su propia área disciplinar y, por último, señalar que existe una menor representatividad en los puestos de mayor responsabilidad laboral, dentro de la institución sanitaria y en la esfera pública.

Al hilo del carácter androcéntrico analizado que ha prevalecido en el ámbito de la salud desde tiempos históricos, cabe resaltar aspiraciones y estrategias por parte de las mujeres que han desarrollado estas profesiones. Dichas estrategias han favorecido el mantenimiento de su seña de identidad como cuidadoras de la salud de un sistema público sanitario, cuya base mayoritaria se nutre de su práctica y experiencia, donde se sustenta la calidad de dicho sistema, uno de los logros más emblemáticos de nuestra democracia. 


\section{Referencias bibliográficas}

Actas del Congreso Internacional de Enfermería (CIE). 2003. Hacia la excelencia mediante la evidencia. Ginebra (Suiza): Consejo Internacional de Enfermeras. Versión en CD-Rom.

Alberdi, Rosa Ma 1998-1999. "Los contextos conceptuales históricos: una propuesta de análisis de la evolución de la profesión enfermera desde los orígenes hasta el siglo XIX”. Híades. Revista de Historia de la Enfermería. 5-6: 63-73.

Álvarez, Carmen. 1998. La mujer como profesional de la medicina en la España del S. XIX. Barcelona: Anthropos.

Arenal, Concepción. Versión facsímil. 1946. El visitador del Pobre. Madrid: E. V Suárez.

Ballarín, Pilar. 2001. La educación de las mujeres en la España contemporánea (siglos $X I X$ y XX). Madrid: Síntesis.

Blanco, Ana Isabel. 2002. Nuevas visiones de la maternidad. León: Universidad de León.

Borderías, Cristina et. al. 1994. Las Mujeres y el trabajo. Madrid: Ikaria.

Cabré, Montserrat y Ortiz, Teresa. 2001. Sanadoras, matronas y médicas en Europa, siglos XII-XX. Barcelona: Icaria.

Capel, Rosa Ma . 1982. El trabajo y la educción de la mujer en España (1900-1930). Madrid: Ministerio de Cultura.

Ceballos, M. 2000. "Los quirófanos, el último bastión masculino". El País, 13 agosto.

Colliére, Marie-Fraçoise. 1993. Promover la vida. Madrid: Interamericana.

De la Rica, Sara y Anghel Brinduesa. 2010. "Diferencias de género en la formación científica: evidencia empírica". Libro Blanco sobre la situación de las mujeres en la ciencia española. Eds. Inés Sánchez de Madariaga, Sara de la Rica y Juan José Dolado. Madrid: Ministerio de Ciencia e Innovación. 19-41.

Domínguez, C. et al. 1993. "Pasado, presente y futuro de la enfermería en el contexto de la salud". Málaga: Servicio de publicaciones de la Diputación Provincial de Málaga 1: 223-231.

Domínguez, Carmen. 1986. Los cuidados y la profesión enfermera en España. Madrid: Pirámide.

Flecha, Consuelo. 2001. "La educación de la mujer según las primeras doctoras en medicina de la Universidad española, año 1982”. Sanadoras, matronas y médicas en Europa. Eds. Montserrat Cabré y Teresa Ortíz. Barcelona: Ikaria. 217-238.

García, Mas et al. 1988. Evaluación de la presencia de la mujer en el sistema sanitario. Madrid: Instituto de la Mujer. 
González, Carmen. 2006. Las cuidadoras. Historia de las matronas, enfermeras y practicantas (1857-1930). Almería: Instituto de Estudios Almerienses.

2007. Asistencia sanitaria, género y cuestión social en Almería (1857-1930). Tesis doctoral. Almería: Servicio de publicaciones Universidad de Almería.

González, Máximo. 2012. "Un incremento del desempleo inversamente proporcional a la seguridad del paciente". Enfermería Facultativa 159: 3.

Harmer \& Henderson, Virginia. 1955. Textbook of the Principles and Practise of Nursing. New-York: Marmillan.

Lillo, M. 2002. “Antropología, género y enfermería contemporánea”. Revista Rol de Enfermería 25, 12: 856-862.

Martínez, Carmen y Ortiz, Teresa. 1998. "Género y profesiones sanitarias. El trabajo de las matronas en Granada en el tránsito del siglo XIX al XX”. La medicina en el S. $X X$. Estudios históricos sobre medicina, sociedad y estado. Eds. J. Castellanos et al. Málaga SEHM: 603-610.

Miqueo, Consuelo et al. 2003. "Del análisis crítico a la autoridad femenina en la ciencia". Feminismo y multidisciplinariedad. Serie Feminismo/s. 1. Coord. Helena Establier. Alicante: Centro de Estudios sobre la Mujer de la Universidad de Alicante: 195-216.

Moreno, Bernardo et al. 2002. "Variables socio-demográficas en el proceso de desgaste profesional de enfermería". Revista Rol de Enfermería 11: 18-26.

Ortiz, Teresa. 1999. "Las matronas y la transmisión de saberes científicos sobre el parto en la España del siglo XIX". Arenal. Revista de historia de las mujeres, 6, 1: 5579.

Ortiz, Teresa. 2006. Medicina, historia y género. 130 años de investigación feminista. Oviedo, KRK ediciones, 2006.

Pizurki, Elena. 1988. Función de las mujeres en la asistencia sanitaria. Ginebra: OMS.

Ramos, María Dolores y Romo, Carmen. 1998. La medida del mundo. Género y usos del tiempo en Andalucía. Sevilla-Málaga: Instituto Andaluz de la Mujer.

Ramos, Ramón. 1990. Cronos dividido. Usos del tiempo y desigualdad entre mujeres y hombres en España. Madrid: Instituto de la Mujer.

Santo-Tomás, Magdalena. 1995. "Historia de la enfermería”. Enfermería Fundamental. Ed. Carmen Fernández. Barcelona: Masson: 3-129.

Siles, José. 1995. Estructura y función social de la Mujer. Alicante: Instituto de Cultura Joan Gil-Albert/Diputación de Alicante.

Vigil, Ma . Dolores. 1996. "La sublevación de las enfermeras". La Correa Feminista 15: 40-41. 